
\title{
25 Research Square \\ Work Engagement and Associated Factors Among Dental Nurses in China
}

\section{Yujing Wang ( 65245913@qq.com)}

School and Hospital of Stomatology, China Medical University, Liaoning Provincial Key Laboratory of Oral Diseases

\section{Yuqin Gao}

School and Hospital of Stomatology, China Medical University, Liaoning Provincial Key Laboratory of Oral Diseases

\section{Yang Xun}

School and Hospital of Stomatology, China Medical University, Liaoning Provincial Key Laboratory of Oral Diseases

\section{Research Article}

Keywords: Work engagement, Job stress, Perceived Social Support, Subjective well-being, Psychological flexibility, Nurse

Posted Date: March 1st, 2021

DOl: https://doi.org/10.21203/rs.3.rs-243167/v1

License: (c) (1) This work is licensed under a Creative Commons Attribution 4.0 International License. Read Full License

Version of Record: A version of this preprint was published at BMC Oral Health on August 16th, 2021. See the published version at https://doi.org/10.1186/s12903-021-01766-y. 


\section{Abstract}

Aim: To identify the level of work engagement among dental nurses in China and explore the correlation between work engagement and psychological characteristics.

Background: Work engagement is affected by many factors, level of work engagement among dental nurses is unknown.

Methods: A cross-sectional questionnaire survey was conducted among 215 dental nurses. The Utrecht Work Engagement Scale (UWES-9), Chinese Nurse Stressors Scale (CNSS), Work-related Acceptance and Action Questionnaire (WAAQ), Multi-Dimensional Scale of Perceived Social Support (MSPSS), and General Well-Being Schedule (GWBS) were applied to measure Chinese nurses' work engagement, job stress, psychological flexibility, perceived social support and subjective well-being respectively. Univariate analysis was used to identify the relationships of work engagement with demographic and psychological characteristics. Hierarchical regression analysis was applied to test the variance in work engagement accounted for by factors related to work engagement in the univariate analysis.

Results: The level of work engagement in Chinese dental nurses was moderate or above. Work engagement was positively associated with perceived social support, psychological flexibility and subjective well-being but negatively correlated with job stress. The hierarchical regression analysis showed that job stress, psychological flexibility and subjective well-being were significantly correlated with work engagement, which explained $36.2 \%$ of the variance in work engagement.

Conclusions: Dental nurses in China had an acceptable level of work engagement in terms of vigour, dedication and absorption. Increased job stress would result in lower work engagement. Nurses who had higher levels of psychological flexibility and subjective well-being also had higher work engagement. We should emphasize the job stress of nurses, strengthen support for organizational culture, create a good work environment and interpersonal relationships, relieve nurses' job stress, improve nurses' levels of subjective well-being, and thus improve nurses' work engagement levels.

\section{Background}

Work engagement is a positive, substantial, work-related mental state, including vigour (i.e., energy), dedication (i.e., involvement and significance) and absorption (i.e., concentration) [1]. Work engagement was found to have a direct impact and promotion on the quality of care [2,3], and many factors, such as organizational and social support [4,5], self-efficacy [6], optimism [7], family [8,9], and different work demands [10], have been shown to have an impact on the level of work engagement. Work engagement is a result of personal learning (i.e., sensibility, reflection) and the work environment [2], with the work environment possibly playing a more important role in work engagement. It has been indicated that engaged employees have less job stress and depression than non-engaged employees [11] and that a high level of work engagement can enhance nurses' job performance, job satisfaction, and emotional health and reduce turnover intention [6]. Since work engagement is important to both organizations and 
individuals, it is necessary to investigate the level of work engagement of nurses in stomatology departments. At present, there are few studies on the work engagement of nurses in stomatology departments.

Good oral health is currently a starting point for people's general health and well-being [12]. As people pay increasing attention to oral health, the quality of oral care is becoming a greater concern. Dental Nurses need to closely cooperate with doctors in the treatment of patients. Nurses are not only cooperate with doctors during operations but also manage equipment materials and patients. They are also oral health educators. They are responsible for the psychological nursing of patients, as well as the prevention and control of infection. The complexity of stomatological materials and the complexity of the maintenance

and use of instruments and equipment increase over time. To prevent nursing errors, dental nurses should be able to concentrate and devote themselves to their work. Some studies have concluded that job stress is positively associated with work engagement [13], while other studies have found the opposite results, i.e., that job stress is negatively associated with work engagement $[14,15]$. The inconsistent results may be due to different occupations, work content, intensities and work requirements, which lead to different levels of job stress. An appropriate amount of stress may promote an increase in work engagement, but once job stress exceeds an individual's tolerance, it will have a negative impact on work engagement. Many psychological characteristics can affect individuals' work engagement. The purpose of this paper is to investigate the work engagement levels of dental nurses in China and identify the influence of psychological characteristics on the level of work engagement through hierarchical regression analysis. This study provides a reference for subsequent intervention studies to improve the work engagement levels of nurses.

\section{Methods}

\section{Design}

A cross-sectional questionnaire survey was conducted among 215 dental nurses in December 2020. All procedures were performed in accordance with relevant guidelines.

\section{Sample and setting}

The research was carried out at the School and Hospital of Stomatology, China Medical University, in December 2020. The study was approved by the Committee on Human Experimentation of China Medical University (2020-22). Nurses from the School and Hospital of Stomatology, China Medical University, participated in the survey. Before the study, an informed consent form was distributed to and collected from nurses. Nurses were included if (1) they had $>1$ working years, (2) they had obtained a nurse qualification certificate, and (3) they provided informed consent. Patients were excluded if (1) they were on maternity leave, (2) they had $>3$ months of sick leave, or (3) they were not engaged in nursing. Questionnaires were sent out via an online QR code with the questionnaire's response time was 20 
minutes. Of the 254 nurses who were sent questionnaires, 215 (84.6\%) responded and completed the questionnaires.

\section{Measurement}

\section{Work engagement}

The 9-item Utrecht Work Engagement Scale (UWES) [16] was used to evaluate the nurses' work engagement. The UWES includes three dimensions: vigour (3 items), dedication ( 3 items), and absorption ( 3 items). Each item is rated on a 7-point scale, where 0 is "never" and 6 is "always". The total score is computed by summing the score of the three dimensions. The ratio of the total score to the item number is the total average score, and the ratio of each dimension score to the item number is the average dimension score. A higher score indicates a higher level of work engagement. The UWES has been proven to have satisfactory reliability and validity in China [17]. The Cronbach's alpha in this study was 0.948 .

\section{Job stress}

We used the Chinese Nursing Stress Scale (CNSS) to measure the dental nurses job stress [18]. The CNSS has been proven to be useful and reliable for evaluating the job stress of nurses and has been widely used in China [19]. It contains 35 items across five dimensions: stress from nursing practice and care (7 items), stress from workload and time allocation (5 items), stress from the work environment and resources ( 3 items), stress from patient care (11 items), and stress from management and relationships (9 items). Each item is rated on a 4-point scale ranging from 1 ("never") to 4 ("almost every day"). The total score ranges from 35 to 140 . A higher score indicates higher job stress. The Cronbach's alpha was 0.960 in this study.

\section{Psychological flexibility}

We used the Work-related Acceptance and Action Questionnaire(WAAQ) [20] to measure the nurses' psychological flexibility in occupational settings. Xu et a/ translated the WAAQ to Chinese in 2018, and it has been proven to be useful and reliable, with a Cronbach's a of 0.920 [21]. The WAAQ includes 7 items rated on a 7-Likert scale from 1 ("never") to 7 ("always"). The total score of the WAAQ ranges from 7 to 49; a higher WAAQ indicates better psychological flexibility, better job acceptance, and greater work activity. The Cronbach's alpha in this study was 0.949 .

\section{Perceived social support}

Zimet et al [22] developed the Multi-Dimensional Scale of Perceived Social Support (MSPSS) in 1988. The MSPSS includes 12 items, with each item rated on a 7-point Likert-type scale, where 1 is "very strongly disagree" and 7 is "very strongly agree". The total score of the MSPSS ranges from 12 to 84 . A higher score indicates better social support and higher satisfaction. The scale contains 3 dimensions: family support (4 items), friend support (4 items) and other support (e.g., relatives and colleagues). The scale emphasizes individual understanding and feelings regarding perceived social support. The Chinese 
version of the MSPSS has been widely used in China and has been proven to be a good measure to evaluate an individual's perceived social support [23].

\section{Subjective well-being}

The General Well-Being Schedule (GWBS) was used to assess nurses' subjective well-being. Duan translated and revised the schedule into Chinese in 1996 [24]. Duan summarized and analysed international research on happiness and formulated a definition of happiness based on three aspects: others' evaluation of individuals, individuals' own emotional experience and self-evaluation. The Chinese version of the GWBS contains 18 items across 6 dimensions: general health ( 2 items), vigour ( 3 items), positive well-being ( 3 items), depression ( 5 items), self-control ( 3 items), and anxiety ( 2 items). Among the 18 items, 4 items are rated on a 5-point Likert-type scale, 10 items are rated on a 6-point Likert-type scale, and the other 4 items are rated on a 0-10 rating scale. The total GWBS score ranges from 14-120. A higher score reflects a higher level of general well-being. The Chinese version of the GWBS has been widely applied in China and has proven to be useful to measure individuals' subjective well-being $[25,26]$. The Cronbach's alpha in this study was 0.881 .

\section{Statistical analyses}

The demographic data are expressed as frequencies and percentages. Psychological characteristics were analysed with descriptive statistics. The one-way ANOVA/t-test was used to compare the work engagement of individuals with different demographic variables. Pearson's correlations were calculated to analyse the relationships of work engagement with job stress, psychological flexibility, perceived social support and subjective well-being. The relationships of the demographic data, job stress, psychological flexibility, perceived social support and subjective well-being to work engagement identified in the univariate analysis were further examined through hierarchical regression. In step one, the demographic data related to work engagement were entered, and the psychological variables such as work engagement, psychological flexibility, perceived social support and subjective well-being were entered in step two.

\section{Results}

\section{Demographic characteristics and level of work engagement}

The characteristics of the nurses and the level of work engagement among different subgroups are displayed in table 1. The work engagement scores differed among nurses with different ages, professional titles and engagement in exercise $(p<0.05)$.

Table 1. Demographic characteristics and level of work engagement among nurses 


\begin{tabular}{|c|c|c|c|c|}
\hline \multirow[t]{2}{*}{ Characteristics } & \multirow[t]{2}{*}{$\mathbf{N}(\%)$} & \multicolumn{3}{|c|}{ Work Engagement } \\
\hline & & Mean (SD) & $t / F$ & $\mathrm{P}$ \\
\hline Gender & & & 0.430 & 0.668 \\
\hline male & $2(0.9)$ & $40.00(19.80)$ & & \\
\hline female & 213(99.1) & $36.83(10.33)$ & & \\
\hline Age & & & 3.743 & $0.006^{*}$ \\
\hline$<30$ years & $106(49.3)$ & 35.88(11.09) & & \\
\hline $31-35$ years & $75(34.9)$ & $36.75(9.34)$ & & \\
\hline $36-40$ years & $22(10.2)$ & $36.55(8.89)$ & & \\
\hline $40-45$ years & $10(4.7)$ & $48.80(7.12)$ & & \\
\hline$>46$ years & $2(0.9)$ & $36.50(0.71)$ & & \\
\hline Marital status & & & 0.384 & 0.701 \\
\hline unmarried or other & $76(35.3)$ & $37.22(9.88)$ & & \\
\hline married & 139(64.7) & $36.65(10.66)$ & & \\
\hline Highest degree & & & 1.087 & 0.339 \\
\hline junior college & $33(15.3)$ & $38.30(12.28)$ & & \\
\hline undergraduate & 178(82.8) & $36.73(9.90)$ & & \\
\hline Master's & $4(1.9)$ & $30.50(14.06)$ & & \\
\hline Exercise & & & -2.011 & $0.046^{*}$ \\
\hline yes & $101(47)$ & $38.36(10.76)$ & & \\
\hline no & 114(53) & $35.53(9.87)$ & & \\
\hline Post & & & -0.880 & 0.380 \\
\hline nurse & 201(93.5) & $36.69(10.37)$ & & \\
\hline head nurse & $14(6.5)$ & $39.21(10.50)$ & & \\
\hline Night shift & $35(16.3)$ & $35.86(10.03)$ & -0.622 & 0.535 \\
\hline Day shift & $180(83.7)$ & $37.05(10.45)$ & & \\
\hline Professional titles & & & 5.299 & $0.006^{*}$ \\
\hline nurse & $39(18.1)$ & $36.38(11.12)$ & & \\
\hline senior nurse & $152(70.7)$ & $35.97(9.92)$ & & \\
\hline
\end{tabular}




\begin{tabular}{|c|c|c|c|c|}
\hline chief nurse & $24(11.2)$ & $43.21(10.14)$ & & \\
\hline Department & & & -0.770 & 0.442 \\
\hline Outpatient & 154(71.6) & $36.51(10.70)$ & & \\
\hline Inpatient & $61(28.4)$ & $37.71(9.51)$ & & \\
\hline Working years & & & 1.102 & 0.384 \\
\hline $1-5$ years & $48(22.3)$ & $35.71(10.75)$ & & \\
\hline $6-10$ years & $100(46.5)$ & $35.93(10.01)$ & & \\
\hline $11-15$ years & $42(19.5)$ & $38.21(11.08)$ & & \\
\hline $16-20$ years & $15(7.0)$ & $37.07(8.46)$ & & \\
\hline$>20$ years & $10(4.7)$ & $45.60(8.36)$ & & \\
\hline Household income & & & 1.208 & 0.309 \\
\hline$<10$ thousand & 78(36.3) & $36.33(11.19)$ & & \\
\hline $11-15$ thousand & $65(30.2)$ & $36.92(9.61)$ & & \\
\hline $16-20$ thousand & $44(20.5)$ & $36.68(9.17)$ & & \\
\hline 20-30 thousand & $18(8.4)$ & $41.33(10.74)$ & & \\
\hline$>30$ thousand & $10(4.7)$ & $33.20(12.30)$ & & \\
\hline
\end{tabular}

${ }^{*} P<0.05$

UWES and average subscale scores in Chinese dental nurses $(\mathrm{N}=215)$.

The average total work engagement score and average dimension scores in the Chinese nurses are shown in Table 2; all average scores were above 4 (out of a total score of 6). The average dedication score (4.14) was higher than the average scores of the other two dimensions, and the absorption score was the lowest (4.02).

Table 2 UWES and average subscale scores in Chinese dental nurses $(\mathrm{N}=215)$ 


\begin{tabular}{|lllll|}
\hline UWES & Item & Min & Max & Mean (SD) \\
\hline Total & 9 & 0.67 & 6 & $4.10(1.15)$ \\
\hline Vigour & 3 & 1.00 & 6 & $4.12(1.14)$ \\
\hline Dedication & 3 & 0.33 & 6 & $4.14(1.23)$ \\
\hline Absorption & 3 & 0.67 & 6 & $4.02(1.26)$ \\
\hline
\end{tabular}

\section{Univariate analysis of the relationships of work engagement with demographic and psychological characteristics $(\mathrm{N}=\mathbf{2 1 5})$.}

Univariate analysis of the relationships of work engagement with demographic and psychological characteristics are displayed in Table 3. Age $(r=0.169, p<0.01)$, professional title $(r=0.143, p<0.05)$, exercise $(r=0.137, p<0.05)$ and working years $(r=0.167, p<0.05)$ were positively correlated with work engagement. The psychological variables, including the CNSS score $(r=-0.440, p<0.01)$, WAAQ score $(r=0.527, p<0.01)$, MSPSS score $(r=0.414, p<0.01)$ and GWBS score $(r=0.451, p<0.01)$, were found to be positively associated with work engagement.

Table 3 Univariate analysis of the relationships between work engagement and other factors $(\mathrm{N}=215)$.

\begin{tabular}{|c|c|c|c|c|c|c|c|}
\hline & Means & SD & UWES & CNSS & WAAQ & MSPSS & GWBS \\
\hline \multicolumn{8}{|l|}{ Categorical r(p) } \\
\hline Age & & & $0.169^{\star \star}$ & 0.071 & 0.050 & 0.034 & 0.060 \\
\hline Professional title & & & $0.143^{*}$ & 0.030 & $0.139^{*}$ & $0.234^{\star *}$ & $0.149^{*}$ \\
\hline Exercise & & & $0.137^{\star}$ & 0.089 & 0.033 & 0.038 & 0.120 \\
\hline Working years & & & $0.167^{\star}$ & -0.003 & 0.066 & 0.056 & 0.005 \\
\hline \multicolumn{8}{|l|}{ Continuous $r(p)$} \\
\hline UWES & 36.86 & 10.369 & 1 & & & & \\
\hline CNSS & 65.03 & 16.563 & $-.440^{\star \star}$ & 1 & & & \\
\hline WAAQ & 38.32 & 8.299 & $.527^{\star \star}$ & $-.385^{\star \star}$ & 1 & & \\
\hline MSPSS & 68.13 & 12.579 & $.414^{\star *}$ & $-.389^{* \star}$ & $.542^{\star \star}$ & 1 & \\
\hline GWBS & 68.87 & 11.848 & $.451^{\star \star}$ & $-.547^{\star \star}$ & $.362^{\star \star}$ & $.439^{\star \star}$ & 1 \\
\hline
\end{tabular}


${ }^{*} P<0.05,{ }^{* \star} P<0.01,{ }^{* \star} P<0.001$ (two-tailed)

\section{Hierarchical regression analysis of work engagement $(\mathrm{N}=215)$.}

A hierarchical regression analysis was performed to test the variance in work engagement accounted for by factors related to work engagement in univariate analysis. Demographic data, job stress, resilience, social support and subjective well-being, which were found to be related to job engagement, were entered into the regression equation. In step one, the demographic data (age, professional title, and exercise) related to work engagement were entered, and then psychological variables such as job stress, psychological flexibility, social support and subjective well-being were entered into the regression in the second step. Job stress, psychological flexibility and subjective well-being were significantly correlated with job engagement, which explained $36.2 \%$ of the variance in work engagement. The results of the hierarchical regression analysis are presented in Table 4.

Table 4 Hierarchical regression analysis of work engagement ( $N=215)$.

\begin{tabular}{|lll|}
\hline WE & Step 1 beta & Step 2 beta \\
\hline Age & 0.073 & 0.066 \\
\hline Professional title & 0.060 & -0.064 \\
\hline Exercise & 0.123 & 0.090 \\
\hline Working years & 0.062 & 0.133 \\
\hline CNSS & & $-0.194^{\star \star}$ \\
\hline WAAQ & & $0.339^{* \star \star}$ \\
\hline WSPSS & & 0.077 \\
\hline GWBS & & $0.182^{* \star}$ \\
\hline F & $2.650^{*}$ & $18.212^{* \star *}$ \\
\hline$R^{2}$ & 0.048 & 0.414 \\
\hline Variance explained & 0.030 & 0.392 \\
\hline$R^{2}$ change & & 0.362 \\
\hline
\end{tabular}

${ }^{*} P<0.05,{ }^{* \star} P<0.01,{ }^{* \star *} P<0.001$ (two-tailed) 


\section{Discussion}

Work engagement is influenced by many factors, such as family [8], personality [27], exercise [28], and diet [29]. A study on work engagement among 3887 rescue workers ( $50 \%$ nurses, $39 \%$ firefighters and $11 \%$ policemen) showed that firefighters had the highest work engagement, while nurses had the lowest work engagement [30], which may be related to the external needs of nurses, the lack of manpower and resources, and conflict with or aggression by patients or their families [31]. A high level of work engagement can enhance nurses' job performance, job satisfaction, and emotional health and reduce turnover intention [6] and has a positive impact on nurses' attitudes towards patients [32]. It has been showed that Chinese nurses [33] had lower work engagement than nurses from other countries [34]. The level of work engagement in Chinese dental nurses in this study was moderate or above, with an average total work engagement score and average subscale scores above 4 (out of a highest possible score of 6 ). The total average score (4.10) was higher than those reported in an international study (4.05) [35] and Simpson's study (4.03) [36]. Among the vigour, dedication, and absorption dimensions, nurses had the highest score for the dedication dimension (4.14), but this score was still lower than those reported in Schaufeli's study (4.28) [35] and Simpson's study (4.37) [36]. The nurses scored the lowest for absorption (4.02), which was higher than that in Schaufeli's international study (3.68) [35].

Work engagement was positively associated with perceived social support, psychological flexibility and subjective well-being but negatively correlated with job stress. A survey of 726 employees in Finland showed that physical and mental health factors were positively correlated with work engagement, while psychosocial risk factors (i.e., anxiety, stress) were negatively correlated with work engagement [28]. Age, professional title, exercise, working years, perceived social support, psychological flexibility, subjective well-being and job stress together explained $39.2 \%$ of the variance in work engagement. Age, professional title, exercise, working years and perceived social support were positively associated with work engagement in the univariate analysis, while the hierarchical regression analysis showed that job stress $(b=-0.194, p<0.01)$, psychological flexibility $(b=0.339, p<0.001)$ and subjective well-being $(b=0.182$, $p<0.01$ ) were significantly correlated with job engagement and explained $36.2 \%$ of the variance in work engagement.

In the European Working Conditions Survey (EWCS 2000), job stress was found to be the second most common work-related health problem [37]. The overall pressure of nurses had a negative impact on mental health [38,39]. Job stress among nurses reduced patient-perceived reliability and assurance, which meant that patient-perceived nursing quality declined [40]. Higher job stress levels had many negative effects, such as reduced satisfaction, burnout, turnover and poor sleep quality. Although Keykaleh et al [41] asserted that nurses' job stress was not correlated with patients' safety, Rainbow et al suggested that job stress could lead to a decline in nurses' performance and increase the safety risk of patients [42]. Although some studies concluded that job stress was positively associated with work engagement [13], the opposite results were found in this study: job stress was negatively associated 
$(b=-0.194, p<0.01)$ with work engagement, similar to other studies $[14,15]$. Health care workers bear more psychological and physical stress than other professionals [38].

Psychological flexibility can be defined as the ability to act according to goals and values in the context of disturbing psychological experience [43]. Employees who exhibited better psychological flexibility were found to be more open when experiencing frustration and difficulties at work and more adaptive to changes at work [37]. A higher level of psychological flexibility was also shown to be associated with greater work engagement and satisfaction [44]. A study of work engagement in 124 residents and 69 experts in five hospitals in the Netherlands reported that residents' work engagement was associated with psychological flexibility, while experts' work engagement was associated with colleague support [45], which indicated that everyone's work engagement may be different and be affected by many factors. The hierarchical regression analysis in this study showed a significant positive correlation $(b=0.339, p<0.001)$ between work engagement and psychological flexibility, which was similar to the findings of other studies [46].

Subjective well-being refers to happiness or satisfaction, which is related to good social relations, work performance and creativity [47]. People feel happiness when they feel more pleasant emotions than unpleasant emotions. Nurses' subjective well-being is very important for both individuals and organizations, as it helps increase job stability and satisfaction [48]. In many studies on subjective wellbeing, work engagement was found to be an intermediary factor [49], which meant that an increasing level of work engagement could indirectly improve an individual's subjective well-being. Subjective wellbeing was found to be moderately related to work engagement among Polish individuals [50]. A study conducted with a sample of 319 full-time in-service kindergarten teachers concluded that subjective wellbeing had a positive association with work engagement [51]. The hierarchical regression analysis in this study showed a significant positive correlation $(b=0.182, p<0.01)$ between work engagement and subjective well-being, similar to other studies [52]. Certain levels of work engagement can enhance subjective well-being, while workaholism is negatively associated with subjective well-being [53].

A significant positive correlation was reported between college teachers' work engagement and students' social support [4]. Providing more support at work can help female nurses achieve balance between family and work and increase work engagement [54]. Providing independent and diverse development opportunities and social support resources can increase employees' work engagement [55]. Although perceived social support was positively correlated with work engagement $(r=0.414, p<0.01)$ in the univariate analysis, it was not positively associated with work engagement $(b=0.077, p>0.05)$ in the hierarchical regression analysis. This inconsistency may be due to perceived social support acting as a mediator between perceived social support and work engagement [56].

\section{Conclusion}

Dental nurses in China had an acceptable level of work engagement in terms of vigour, dedication and absorption. work engagement was positively associated with perceived social support, psychological 
flexibility and subjective well-being but negatively correlated with job stress, which implied that increased job stress resulted in lower work engagement. Nurses who had higher levels of psychological flexibility and subjective well-being also had higher work engagement. Job stress, psychological flexibility and subjective well-being were significantly correlated with job engagement, which explained $36.2 \%$ of the variance in work engagement. We should emphasize the job stress of nurses, strengthen support for organizational culture, create a good work environment and interpersonal relationships, relieve nurses' job stress, improve nurses' levels of subjective well-being, and thus improve nurses' work engagement levels.

\section{Declarations}

Authors details: Yujing Wang ${ }^{1 *}$, Yuqin Gao ${ }^{1}$,Yang Xun ${ }^{1}$

${ }^{1}$ Department of Nursing, School and Hospital of Stomatology, China Medical University, Liaoning Provincial Key Laboratory of Oral Diseases, Shenyang, People's Republic of China.

* corresponding author

\section{Authors' contributions}

YJW and YQG were responsible for the research design. YJW was engaged in data processing, data analysis and wrote the manuscript. YX was responsible for the production, distribution and collection of electronic questionnaires. All authors have reviewed the manuscript and given final approval of the version to be published.

\section{Funding}

This research did not receive any specific grant from funding agencies in the public, commercial, or notfor-profit sectors.

\section{Ethics approval and consent to participate}

All procedures were performed in accordance with relevant guidelines. All study materials were approved by Committee on Human Experimentation of China Medical University (2020-22). Informed consent form were filled in by paper questionnaire and collected.

\section{Acknowledgments}

The authors would like to thank all the nurses that participated in the study.

\section{Availability of data and materials}

Data and materials are available from the corresponding author on reasonable request.

\section{Competing interests}


All authors have no conflicts of interest.

\section{Consent for publication}

Not applicable.

CorrespondingAuthor: Yujing Wang

Correspondence Address: Department of Nursing School and Hospital of Stomatology, China Medical University, Liaoning Provincial Key Laboratory of Oral Diseases, Shenyang, 110002, People's Republic of China.

E-mail:65245913@qq.com

Tel: $86-13889838770$

\section{Authors' contributions:}

YJW and YQG were responsible for the research design. YJW was engaged in data processing, data analysis and wrote the manuscript. YX was responsible for the production, distribution and collection of electronic questionnaires. All authors have reviewed the manuscript and given final approval of the version to be published.

\section{Funding:}

NO fundings.

\section{Ethics approval and consent to participate}

All study materials were approved by Committee on Human Experimentation of China Medical University (2020-22). Informed consent form were filled in by paper questionnaire and collected.

\section{References}

[1] Bakker AB, Demerouti E, Sanz-Vergel Al. Burnout and work engagement: The JD-R approach. ANNU REV ORGAN PSYCH.2014; 1(1):389- 411.

https://doi.org/10.1146/annurev-orgpsych-031413-091235

[2] García-Sierra R, Fernández-Castro J, Martínez-Zaragoza F. Work engagement in nursing: an integrative review of the literature. J Nurs Manag.2016; 24(2): E101-11.

https://doi.org/ 10.1111/jonm.12312. 
[3] Van Bogaert P, Clarke S, Willems R, Mondelaers M. Staff engagement as a target for managing work environments in psychiatric hospitals: implications for workforce stability and quality of care. J Clin Nurs.2013;22(11-12):1717- 1728.

https://doi.org/ 10.1111/j.1365-2702.2012.04341.x

[4] Martini M, Guidetti G, Viotti S, Loera B, Converso D. Sometimes It Drains,

Sometimes It Sustains: The Dual Role of the Relationship with Students for University Professors.

Biomed Res Int.2019; 2019:9875090. https://doi.org/ 10.1155/2019/9875090.

[5] Gupta V, Agarwal UA, Khatri N. The relationships between perceived organizational support, affective commitment, psychological contract breach, organizational citizenship behaviour and work engagement. J Adv Nurs. 2016;72(11):2806-2817.

https://doi.org/ 10.1111/jan.13043.

[6] Salanova M, Lorente L, Chambel MJ, Martínez IM. Linking transformational

leadership to nurses' extra-role performance: the mediating role of self-efficacy and work engagement. $J$ Adv Nurs. 2011;67 (10)凶2256- 2266.

https://doi.org/ 10.1111/j.1365-2648.2011.05652.x.

[7] Garrosa E, Moreno-Jiménez B, Rodríguez-Muñoz A, Rodríguez-Carvajal R.. Role

stress and personal resources in nursing: a cross-sectional study of burnout and engagement. Int J Nurs Stud. 2011;48(4):479-89. https://doi.org/10.1016/j.ijnurstu.2010.08.004.

[8] Lu CQ, Siu OL, Chen WQ, Wang HJ. Family mastery enhances work engagement in Chinese nurses: A cross-lagged analysis. J Vocat Behav. 2011;78(1):100-109. https://doi.org/10.1016/j.jvb.2010.07.005.

[9] Rofcanin Y, Las Heras M, Bakker AB. Family supportive supervisor behaviors and

organizational culture: Effects on work engagement and performance. J Occup Health Psychol. 2017;22(2):207-217. https://doi.org/ 10.1037/ocp0000036.

[10] Mijakoski D, Karadzinska-Bislimovska J, Basarovska V, Montgomery A, Panagopoulou E, Stoleski S, Minov J. Burnout, Engagement, and Organizational Culture: Differences between Physicians and Nurses. Open Access Maced J Med Sci. 2015;3(3):506-13.

https://doi.org/ 10.3889/oamjms.2015.091.

[11] Schaufeli WB, Bakker AB. Job demands, job resources, and their relationship with burnout and engagement: a multi-sample study. Journal of Organizational Behavior. 2004;25『293- 315. 
[12] Fiorillo L. Oral Health: The First Step to Well-Being. Medicina (Kaunas). 2019;55(10):676. https://doi.org/ 10.3390/medicina55100676.

[13] Inoue A, Kawakami N, Tsutsumi A, Shimazu A, Miyaki K, Takahashi M, Kurioka S, Eguchi H, Tsuchiya M, Enta K, Kosugi Y, Sakata T, Totsuzaki T. Association of job demands with work engagement of Japanese employees: comparison of challenges with hindrances (J-HOPE). PLoS One.2014; 9(3): e91583. https://doi.org/ 10.1371/journal.pone.0091583.

[14] Klein CJ, Weinzimmer LG, Cooling M, Lizer S, Pierce L, Dalstrom M. Exploring burnout and job stressors among advanced practice providers. Nurs Outlook. 2020;68(2):145-154. https://doi.org/ 10.1016/j.outlook.2019.09.005.

[15] Mauno S, Ruokolainen M, Kinnunen U, De Bloom J. Emotional labour and work engagement among nurses: examining perceived compassion, leadership and work ethic as stress buffers. J Adv Nurs. 2016;72(5):1169-81. doi: 10.1111/jan.12906.

[16] Schaufeli WB, Bakker AB, Salanova M. The measurement of work engagement with a short questionnaire: A cross-national study. Educ Psychol Meas. 2006; 66:701-16.

[17] Zhu Y, Liu C, Guo B, Zhao L, Lou F. The impact of emotional intelligence on work engagement of registered nurses: the mediating role of organisational justice. Journal of Clinical Nursing. 2015; 24(1516):2115-2124. doi: 10.1111/jocn.12807.

[18] Li XM, Liu YJ. Job Stressors and Burnout among Staff Nurses. Chinese Journal of Nursing. 2000; 35 (11), 645- 649 in Chinese.

[19] Yao X, Shao J, Wang L, Zhang J, Zhang C, Lin Y. Does workplace violence, empathy, and communication influence occupational stress among mental health nurses? Int J Ment Health Nurs. 2020 Aug 17. doi: 10.1111/inm.12770.

[20] Bond FW, Lloyd J, Guenole N. The work-related acceptance and action questionnaire: Initial psychometric findings and their implications for measuring psychological flexibility in specific contexts. $J$ Occup Organ Psychol. 2013; 86囚331-347.

[21] Xu XH, Liu XY, Ou MJ, Xie CJ, Chen YY. Psychological flexibility of nurses in a cancer

hospital『preliminary validation of a Chinese version of the work-related acceptance and action questionnaire. Asia Pac J Oncol Nurs. 2018;5(1)囚83-90.

doið10.4103/apjon.apjon_62_17.

[22] Zimet GD, Dahlem NW, Zimet SG, Farley GK. The multidimensional scale of perceived social support. Journal of personality assessment. 1988; 52:30-41. 
[23] Liu L, Yang YL, Wang ZY, Wu H, Wang Y, Wang L. Prevalence and positive correlates of posttraumatic stress disorder symptoms among Chinese patients with hematological malignancies: a cross-sectional study. PLoS One. 2015;10(12): e0145103.

doi: 10.1371/journal.pone.0145103.

[24] Duan JH. Results and analysis of the general well-being schedule in Chinese college students. China J Clin Psychol. 1996; 4(1):56-7 in Chinese.

[25] Hua J, Zhu LP, Du WC, Du L, Luo T, Wu ZC. Infant's sex, birth control policy and postpartum wellbeing: a prospective cohort study in Shanghai, China. BMJ Open. 2016; 6(10):e012207.doi: 10.1136/bmjopen-2016-012207.

[26] Wang Q, Liu H, Ren Z, Xiong W, He M, Li N, Fan X, Guo X, Li X, Shi H, Zha S, Zhang X. The Associations of Family Functioning, General Well-Being, and Exercise with Mental Health among EndStage Renal Disease Patients. Psychiatry Investig. 2020; 17(4):356-365. doi: 10.30773/pi.2019.0204.

[27] Smith CE, Barratt CL, Hirvo A. Burned out or engaged at work? The role of self-regulatory personality profiles. Stress Health. 2020 Dec 16. doi: 10.1002/smi.3015.

[28] Veromaa V, Kautiainen $\mathrm{H}$, Korhonen PE. Physical and mental health factors associated with work engagement among Finnish female municipal employees: a cross-sectional study. BMJ Open. 2017; 7(10):e017303. doi: 10.1136/bmjopen-2017-017303.

[29] Amano H, Fukuda Y, Baden MY, Kawachi I. Is work engagement associated with healthier dietary patterns? A cross-sectional study. J Occup Health. 2020; 62(1): e12149.

doi: 10.1002/1348-9585.12149.

[30] Sinval J, Marques-Pinto A, Queirós C, Marôco J. Work Engagement among Rescue Workers: Psychometric Properties of the Portuguese UWES.Front Psychol. 2018;8:2229. doi: 10.3389/fpsyg.2017.02229.

[31] Lin TC, Lin HS, Cheng SF, Wu LM, Ou-Yang MC. Work stress, occupational burnout and depression levels: a clinical study of paediatric intensive care unit nurses in Taiwan. J Clin Nurs.2016; 25, 11201130. doi: 10.1111/jocn.13119.

[32] Schultz ASH, Hossain S, Johnson JL. Modeling influences on acute care nurses' engagement in tobacco use reduction. Res Nurs Health.2009; 32(6):621-633. doi: 10.1002/nur.20349.

[33] Wang XX, Liu L, Zou FT, Hao JH, Wu H. Associations of Occupational Stressors, Perceived Organizational Support, and Psychological Capital with Work Engagement among Chinese Female Nurses. Biomed Res Int. 2017; 2017:5284628. doi: 10.1155/2017/5284628. 
[34] Petrović IB, Vukelić M, Čizmić S. Work Engagement in Serbia: Psychometric Properties of the Serbian Version of the Utrecht Work Engagement Scale (UWES). Front Psychol. 2017; 8:

1799. doi: 10.3389/fpsyg.2017.01799.

[35] Schaufeli W, Bakker A. The measurement of work engagement with a short questionnaire. Educ Psychol Meas. 2006; 66(4):701-15.

[36] Simpson MR. Predictors of Work Engagement Among Medical-Surgical Registered Nurses. West J Nurs Res. 2009; 31(1):44-65. doi: 10.1177/0193945908319993.

[37] Ramaci T, Bellini D, Presti G, Santisi G. Psychological Flexibility and Mindfulness as Predictors of Individual Outcomes in Hospital Health Workers.

Front Psychol. 2019; 10:1302. doi: 10.3389/fpsyg.2019.01302.

[38] Chen CH, Wang J, Yang CS, Fan JY. Nurse practitioner job content and stress effects on anxiety and depressive symptoms, and self-perceived health status. J Nurs Manag. 2016; 24(5):695-704. doi: $10.1111 /$ jonm.12375.

[39] Kowalczuk K, Krajewska-Kułak E, Sobolewski M. The Effect of Subjective Perception of Work in Relation to Occupational and Demographic Factors on the Mental Health of Polish Nurses.Front Psychiatry. 2020; 11:591957. doi: 10.3389/fpsyt.2020.591957.

[40] Teng $\mathrm{Cl}$, Hsiao FJ, Chou TA. Nurse-perceived time pressure and patient-perceived care quality. J Nurs Manag. 2010;18(3): 275-84.doi: 10.1111/j.1365-2834.2010.01073.x.

[41] Keykaleh MS, Safarpour H, Yousefian S, Faghisolouk F, Mohammadi E, Ghomian Z. The Relationship between Nurse's Job Stress and Patient Safety. Open Access Maced J Med Sci. 2018; 6(11):2228-2232. doi: 10.3889/oamjms.2018.351.

[42] Rainbow JG, Drake DA, Steege LM. Nurse Health, Work Environment, Presenteeism and Patient Safety. West J Nurs Res. 2020; 42(5):332-339. doi: 10.1177/0193945919863409.

[43] Hayes SC, Luoma JB, Bond FW, Masuda A, Lillis J. Acceptance and commitment therapy: Model, processes and outcomes. Behav Res Ther. 2006; 44(1):1-25.

doi: 10.1016/j.brat.2005.06.006.

[44] Bond FW, Bunce D. The role of acceptance and job control in mental health, job satisfaction, and work performance. J Appl Psychol. 2003; 88(6):1057-67.

doi: 10.1037/0021-9010.88.6.1057.

[45] Solms L, van Vianen AEM, Theeboom T, Koen J, de Pagter APJ, de Hoog M; Challenge \& Support Research Network. Keep the fire burning: a survey study on the role of personal resources for work 
engagement and burnout in medical residents and specialists in the Netherlands. BMJ Open. 2019; 9(11): e031053. doi: 10.1136/bmjopen-2019-031053.

[46] Solms L, van Vianen AEM, Theeboom T, Koen J, de Pagter APJ, de Hoog M; Challenge \& Support Research Network. Keep the fire burning: a survey study on the role of personal resources for work engagement and burnout in medical residents and specialists in the Netherlands. BMJ Open. 2019; 9(11): e031053. doi: 10.1136/bmjopen-2019-031053.

[47] Diener E, Oishi S, Tay L. Advances in subjective well-being research. Nat Hum Behav. 2018; 2(4):253260. doi: 10.1038/s41562-018-0307-6.

[48] Hwang E. Effects of the organizational culture type, job satisfaction, and job stress on nurses' happiness: A cross-sectional study of the long-term care hospitals of South Korea. Jpn J Nurs Sci. 2019; 16(3):263-273. doi: 10.1111/jjns.12235.

[49] Rusu PP, Colomeischi AA. Positivity Ratio and Well-Being Among Teachers. The Mediating Role of Work Engagement. Front Psychol. 2020; 11:1608. doi: 10.3389/fpsyg.2020.01608.

[50] Piotrowski A, Sygit-Kowalkowska E, Hamzah I. Work Engagement among Prison Officers. The Role of Individual and Organizational Factors in the Polish and Indonesian Penitentiary Systems. Int J Environ Res Public Health. 2020; 17(21):8206. doi: 10.3390/ijerph17218206.

[51] De Stasio S, Benevene P, Pepe A, Buonomo I, Ragni B, Berenguer C. The Interplay of Compassion, Subjective Happiness and Proactive Strategies on Kindergarten Teachers' Work Engagement and Perceived Working Environment Fit. Int J Environ Res Public Health. 2020; 17(13):4869. doi: 10.3390/ijerph17134869.

[52] van den Berg JW, Mastenbroek NJJM, Scheepers RA, Jaarsma ADC. Work engagement in health professions education. Med Teach. 2017; 39(11):1110-1118. doi: 10.1080/0142159X.2017.1359522.

[53] Shimazu A, Schaufeli WB. Is workaholism good or bad for employee well-being? The distinctiveness of workaholism and work engagement among Japanese employees. Ind Health.2009; 47(5):495-502. doi: 10.2486/indhealth.47.495.

[54] Okada N, Yabase K, Kobayashi T, Okamura H. Do multiple personal roles promote working energetically in female nurses? A cross-sectional study of relevant factors promoting work engagement in female nurses. Environ Health Prev Med. 2019; 24(1):56. doi: 10.1186/s12199-019-0810-z.

[55] Boelhouwer IG, Vermeer W, van Vuuren T. Work Ability, Burnout Complaints, and Work Engagement Among Employees With Chronic Diseases: Job Resources as Targets for Intervention? Front Psychol. 2020; 11:1805. doi: 10.3389/fpsyg.2020.01805.

[56] Xi YH, Xu YY, Wang Y. Too-Much-Of-A-Good-Thing Effect of External Resource Investment-A Study on the Moderating Effect of Psychological Capital on the Contribution of Social Support to Work 
Engagement. Int J Environ Res Public Health. 2020; 17(2):437. doi: 10.3390/ijerph17020437. 